\title{
Estudio y análisis del aprendizaje y la enseñanza en la educación superior
}

\author{
Violeta Hidalgo ${ }^{1}$, Juan Arias ${ }^{2}$, Rafael Martín ${ }^{2}$ y Juan Ángel Contreras ${ }^{2}$ \\ ${ }^{1}$ Departamento de Tecnología de los Computadores y de las Comunicaciones. Universidad \\ de Extremadura, España | vhidalgo@unex.es | https://orcid.org/0000-0001-6019-8336 \\ ${ }^{2}$ Departamento de Ingeniería de Sistemas Informáticos y Telemáticos, Universidad de \\ Extremadura, Centro Universitario de Mérida, España | jarias@unex.es; rmmartin@unex.es \\ jaconvas@unex.es; | https://orcid.org/0000-0003-2605-3736;; https://orcid.org/0000-0002- \\ 9710-4097; https://orcid.org/0000-0002-2180-1339
}

Resumen: El presente artículo muestra el resultado de la investigación realizada durante el curso 2020/21, en la asignatura de Seguridad de la Información, impartida en Educación Superior, en el cuarto curso del Grado en Ingeniería Telemática en Telecomunicación y también en el Grado en Ingeniería Informática en Tecnologías de la Información, en el Centro Universitario de Mérida de la Universidad de Extremadura. La motivación de la investigación fue realizar un seguimiento de la docencia, de forma no invasiva que sirva de ayuda en e proceso de enseñanza/aprendizaje, mientras que los alumnos no sienten que están siendo evaluados, minimizando el estrés que producen los exámenes, e intentando automatizar el proceso de evaluación del aprendizaje de forma que el docente disponga de una herramienta de uso fácil y rápido que le dé información sobre la evolución en la estructura mental de los alumnos, de forma online. Las bases del estudio que presentamos son diferentes investigaciones sobre redes asociativas pathfinder y conceptos nucleares.El proceso de investigación se desarrolló en varias etapas. El primer paso consistió en obtener el conocimiento previo de los alumnos con la ayuda de software creado para tal fin. Con los datos obtenidos se crearon las redes asociativas pathfinder de cada estudiante para conocer su estructura mental. Se tomaron datos en varios momentos del curso docente, para comprobar la evolución del proceso de enseñanza-aprendizaje y mediante redes asociativas pathfinder se representaron de forma gráfica las estructuras mentales de los alumnos pudiendo observar la evolución del aprendizaje durante el periodo docente.

Palabras clave: Conceptos Nucleares; Educación Superior; Redes Asociativas Pathfinder.

\section{Study and Analysis of Learning and Teaching in Higher Education}

Abstract: This article shows the result of the research carried out during the 2020/21 academic year, in the subject of Information Security, taught in Higher Education, in the fourth year of the Degree in Telematics Engineering in Telecommunications and also in the Degree in Computer Engineering in Information Technology, at the University Center of Mérida of the University of Extremadura. The motivation of the research was to monitor the teaching, in a non-invasive way that helps in the teaching / learning process, while the students do not feel that they are being evaluated, minimizing the stress produced by the exams, and trying to automate the learning assessment process so that the teacher has a quick and easy-to-use tool that provides information on the evolution of the students' mental structure, online. The bases of the study that we present are different investigations on pathfinder associative networks and nuclear concepts. The research process developed in several stages. The first step consisted in obtaining the prior knowledge of the students with the help of software created for this purpose. With the data obtained, the associative pathfinder networks of each student were created to know their mental structure. Data were collected at various moments of the teaching course, to verify the evolution of the teaching-learning process and through associative pathfinder networks, the mental structures of the students were graphically represented, being able to observe the evolution of learning during the teaching period.

Keywords: Nuclear concepts; Higher education; Pathfinder Associative Networks. 


\section{Introducción}

La idea de la investigación que presentamos trata de cómo se produce el aprendizaje a partir de los cambios en las estructuras cognitivas de los alumnos.

Numerosos estudios nos avalan, la base principal fue (Arias, Contreras, Hidalgo y Martín, 2019), que compara la estructura mental de los alumnos y la del profesor, analizando la similaridad que existe entre la red media de las Redes Asociativas Pathfinder, (Arias, Contreras, Hidalgo y Martín, 2019), (Schvaneveldt, 1989a), en adelante RAP, de los alumnos y la del profesor. A través de las RAP, se representaron las estructuras cognitivas respecto del tema a evaluar, concluyendo que un profesor puede obtener información del aprendizaje que reciben sus alumnos mientras imparte un determinado tema y describiendo un método de fácil utilización por parte de los profesores, con el que rápidamente y en tiempo real, se puede comprobar si las estructuras cognitivas de sus alumnos comienzan a acercarse a la red de la ciencia (Arias, 2008), (Hidalgo, Arias, Casas, Luengo y Castillo, 2008) demostrando así el aprendizaje del tema impartido.

\subsection{La red de la Ciencia.}

La red de la ciencia es una RAP que representa información que el profesor quiere transmitir, incluye los conceptos que según el equipo docente son más importantes del tema de estudio, así como su relación, (Contreras, Arias, Luengo y Casas, 2015). Con estos conceptos se crea una tabla bidimensional (matriz de porcentajes), base de la RAP de la red de la ciencia, con la que se comprobará el cambio en la estructura mental de los alumnos, para demostrar el aprendizaje de la materia impartida en la clase.

Como hemos comentado anteriormente, para la elección de los conceptos a evaluar, nos apoyamos en (Contreras, Arias, Luengo y Casas, 2015) que describe cómo elegir los conceptos importantes de un tema de estudio, Contreras, siguiendo la Teoría de los Conceptos Nucleares (Casas, 2002), (Casas y Luengo, 2013) y (Arias, Contreras, Hidalgo y Martín, 2019), obtiene los conceptos importantes, los nucleares, y los fundamentales a partir del índice de nuclearidad. Diferenciando cuantitativamente los conceptos nucleares de los conceptos no nucleares, gracias al índice de nuclearidad completo.

Según (Schvaneveldt, 1989a, 1989b), las RAP que presenten un valor de coherencia menor a 0,15 son redes referentes de datos fallidos. Puede ser debido a diferentes causas, como un error en la entrada de los datos, que los datos no están ordenados de la misma manera, o el formato es erróneo.

La coherencia se calcula a partir de valores de proximidad del conjunto de datos, basándose en la idea de que la distancia entre cada par de conceptos se puede predecir a partir de las distancias de los mismos con el resto de conceptos del conjunto.

Con los datos procedentes de los alumnos, la coherencia ofrece las correlaciones con el grado de aprendizaje.

Los estudios anteriores se basaron en la teoría de los conceptos nucleares, (TCN), de (Casas, 2002) que explica y analiza el aprendizaje de forma distinta a como lo hacen otras teorías de referencia, como las de (Ausubel, Novak y Hanesian, 1978) (Piaget, 1978). Según Casas, se produce aprendizaje en los alumnos al enlazar conceptos concretos de su estructura cognitiva con conceptos nuevos. El conocimiento se representa en pequeñas estructuras, relativamente estables, de elementos relacionados entre sí para cada concepto y se organiza en forma de redes interrelacionadas.

Estos conceptos difieren para cada persona, se trata de conceptos que llaman la atención del alumno y por distintos motivos destacan en su mente. Según Casas, éstos son conceptos nucleares, base sobre la que se forma el conocimiento. 
La estructura creada en este proceso de aprendizaje es diferente para cada alumno, depende de su estructura cognitiva previa, se forma una relación de conceptos que nos puede ayudar a comprender el modo de aprendizaje dado que la estructura cognitiva organizada alrededor de un concepto se hace más sencilla conforme aumenta el conocimiento, Casas lo denomina "senderos de mínimo coste".

La idea es que cuanto mejor se conoce la materia del concepto estudiado, la estructura formada por las relaciones en torno a este concepto es más sencilla dando lugar a RAP más simples, al adquirir una visión global del tema.

La TCN utiliza las Redes Asociativas Pathfinder para analizar la estructura cognitiva del alumno, además de interpretar las diferencias en el desarrollo de los alumnos. Permite el desarrollo de estudios que tengan en cuenta las características del aprendizaje o la estructuración del conocimiento en distintos entornos, áreas de conocimiento, en distintas personas.

De todo lo expuesto anterior surge la investigación que mostramos en este artículo.

\section{Objetivos}

El objetivo de la investigación es determinar un método para que el profesor pueda obtener en tiempo real y de forma no invasiva información acerca de si los alumnos asimilan los contenidos impartidos.

\section{Experimentación}

El presente artículo es fruto de la experiencia realizada durante el primer semestre del curso 2020/2021, en la Universidad de Extremadura, a través de la asignatura Seguridad de la información (Arias, 2020), Área de Ingeniería Telemática de la Universidad de Extremadura. Es una asignatura de tercer curso, del Grado en Ingeniería Telemática en Telecomu-nicación (GITT) y del Grado en Ingeniería Informática en Tecnologías de la Información (GIITI). Tiene una extensión de 6 créditos, divididos en 3,8 créditos de teoría y 2,2 de prácticas, y en este curso cuenta con 32 alumnos matriculados, los cuáles realizan esta experiencia por primera vez.

El temario teórico de la asignatura trata temas como seguridad digital, criptografía, etc., el tema elegido por el equipo de investigación fue "Criptografía moderna" para llevar a cabo la experiencia, y de este tema se eligieron 4 conceptos. Esta elección se debe a que según estudios previos de investigación del grupo Ciberdidact, (Arias, Contreras, Hidalgo y Martín, 2019) aconsejan que no se consideren más de 6 o 7 conceptos a evaluar en cada muestra o toma de información.

El motivo es que en el momento de realizar las comparaciones entre pares de conceptos, la cantidad de pares de relaciones aumenta y hace que los individuos que realizan el test puedan relacionar los conceptos sin el interés necesario. En ese caso, la coherencia de sus redes se debilitaría y los datos no resultarían válidos (Schvaneveldt, 1989). Los conceptos seleccionados son los siguientes:

- Cifrado en flujo: "Un cifrado en flujo es un criptosistema que aplica una función de transformación variable a elementos individuales del texto en claro.", (Martínez, 2016).

- Cifrado en bloque: "Un cifrado en bloque es un criptosistema que aplica una función de transformación fija a bloques del texto en claro. El cifrado en bloque es el que opera sobre el texto en claro en grupos de elementos de longitud fija relativamente grandes (de 64, 128 o 256 bits, por ejemplo), llamados bloques, aplicándoles una función de transformación invariante, es decir, se aplica siempre la misma función; por lo que se dice que no poseen memoria, en referencia a que se guarde un estado del que dependa la transformación, como sucede en un cifrado en flujo, (Martínez, 2016). 
- Registros de desplazamientos: "Un registro de desplazamiento es un circuito digi-tal secuencial (es decir, que los valores de sus salidas dependen de sus entradas y de los valores anteriores) consistente en una serie de biestables, generalmente de tipo $D^{1}$, conectados en cascada, que basculan de forma sincrónica con la misma señal de reloj. Según las conexiones entre los biestables, se tiene un desplazamiento a la izquierda o a la derecha de la información almacenada."("Registro de desplazamiento - Wikipedia, la enciclopedia libre," n.d.).

- Generadores de secuencias: "Son algoritmos determinísticos que a partir de una clave corta (128 bits), conocida por el emisor y el receptor, generan simultáneamente una determinada secuencia de la longitud deseada. (Centro Criptológico Nacional. Gobierno de, 2017).

Una vez determinados los conceptos, se preparó el servidor Meba (Arias, 2017) para la toma de datos, los alumnos realizaron el test pathfinder, y nuestro servidor Meba nos permi-tió obtener los datos en tiempo real.

\subsection{Descripción del Servidor Meba.}

El servidor Meba trabaja con conceptos y alumnos. Los conceptos se agrupan en temas y los alumnos se organizan en grupos, además, se relaciona cada grupo con el tema que se va a evaluar, dando lugar al conjunto de datos: tema, conceptos, grupo y alumnos.

Servidor MeBa o 4022/2021 19:21:17

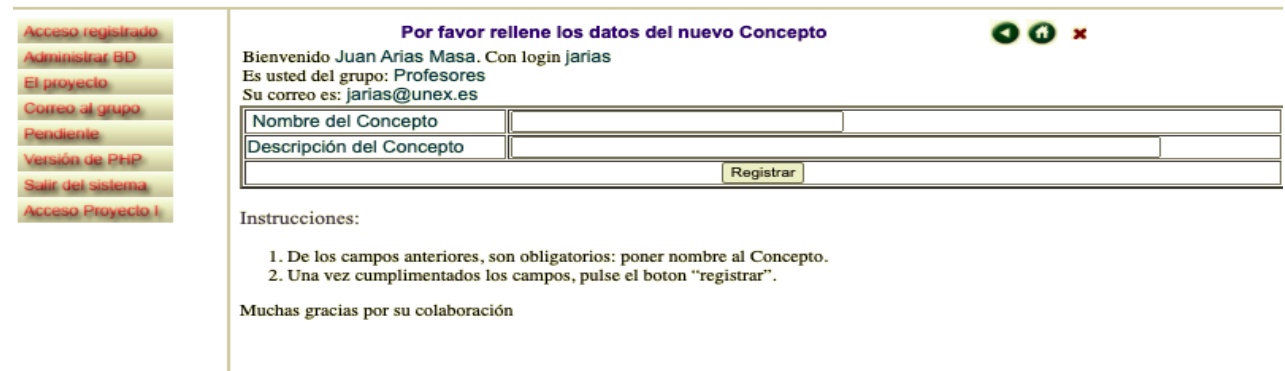

Fig. 1. Pantalla para añadir conceptos en el servidor Meba.

Por otro lado, en Meba existen tres niveles de jerarquía de usuarios registrados con "login" y "clave". Estos tres grupos son:

- Supervisores: los usuarios de este grupo se encargan de coordinar todo el servidor y todos los procesos, tienen acceso completo al sistema. Habitualmente, sólo existen dos usuarios supervisores, uno que es el principal y otro que es de reserva y ambos tienen incompatibilidad de acceso simultaneo al sistema, es decir, el acceso de ambos es excluyente. Igualmente, su principal misión es poder añadir nuevos usuarios al grupo de profesores.

- Profesores: este grupo es el encargado de añadir nuevos alumnos, nuevos temas, nuevos conceptos, etc. Son los que planifican las encuestas de proximidad de con-ceptos, relacionando y conjuntando alumnos en grupos, conceptos en temas, en definitiva, un usuario de este grupo es el que va a posibilitar la toma de datos por parte del servidor Meba de una determinada encuesta de proximidad a un grupo de alumnos. Finalmente, el usuario de este grupo será el que obtenga la infor-mación aportada por los alumnos.

\footnotetext{
${ }^{1}$ Se conocen por el nombre de biestables de datos o seguidores. Tienen una única entrada $\mathrm{D}$, que es copiada en el interior del bi estable en los instantes de sincronismo.
} 
- Alumnos: los usuarios de este grupo son los que realizan las encuestas de proximidad de conceptos.

En consecuencia, será el profesor el responsable de preparar en el servidor Meba la información necesaria para que pueda tener lugar una toma de datos, comenzando por introducir los conceptos a evaluar mediante una interface similar al mostrado en la Fig. $\mathbf{1}$ donde se añade el nombre del concepto y, de opcionalmente, una descripción del mismo.

Los conceptos se agrupan en temas, a través de una interface como el de la Fig. 2. En este caso los datos del tema son el nombre o título del tema, la URL donde se puede encontrar información sobre dicho tema, el email de la persona responsable del tema, y una des-cripción del tema tratado.

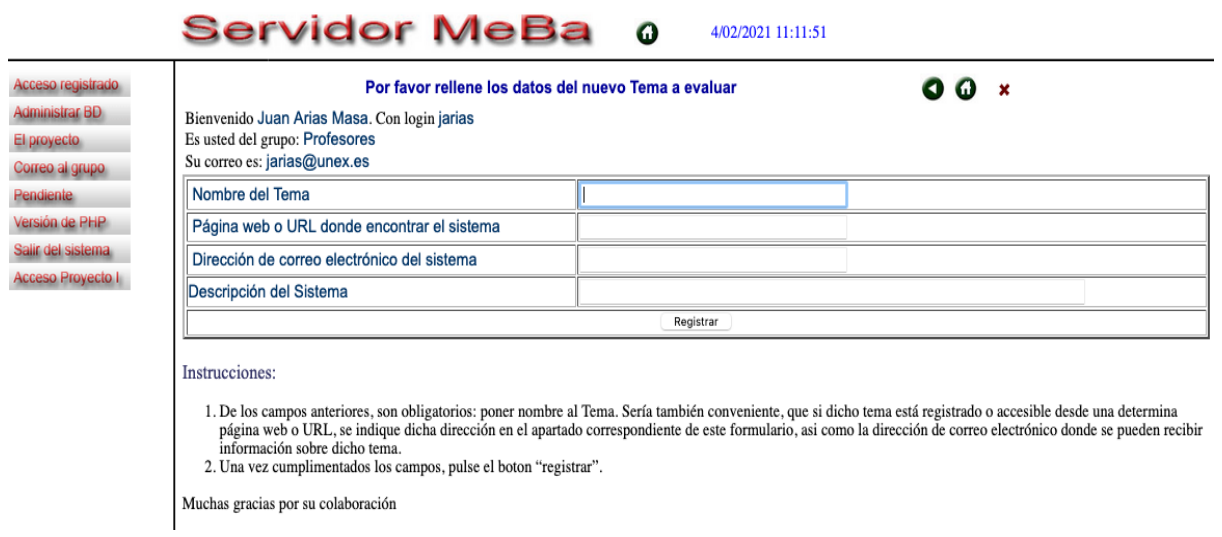

Fig. 2. Pantalla para añadir un nuevo tema en el servidor Meba.

El tercer elemento importante en la toma de datos son los usuarios alumnos, que se añaden en una interface como el mostrado en la Fig. 3. De todos los datos que se pueden incluir, los únicos datos imprescindibles son el "login" y la "clave", el resto, aunque son optativos para el sistema, es muy recomendable completarlos. Igualmente indicamos que dichos datos también pueden ser importados desde un fichero tipo CSV, u otros formatos.

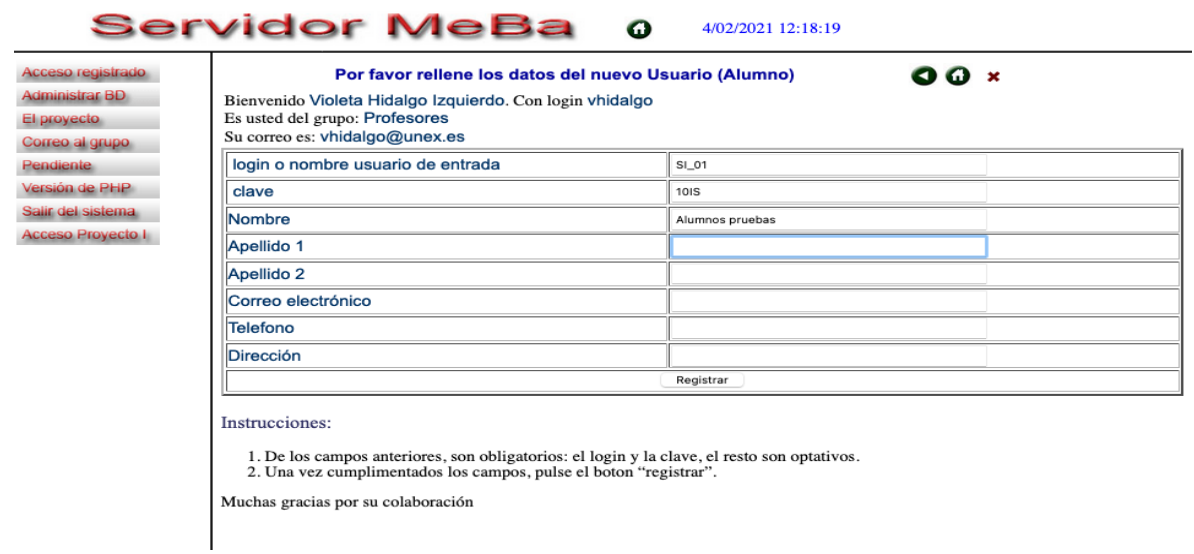

Fig. 3. Pantalla para añadir un nuevo alumno en el servidor Meba.

La toma de datos se realiza ONLINE y en tiempo real a través de Meba, donde a cada alumno se le mostrarán los conceptos de dos en dos, como el mostrado en el ejemplo de la Fig. 4, para que pueda elegir cuál es la relación de proximidad que él estima entre los dos conceptos que le aparecen en la pantalla. 
La citada elección del alumno es posible clicando en la zona del triángulo que el considere para cada uno de los pares, si los considera muy relacionados deberá clicando más a la derecha y si considera que están alejados podrá clicar más a la izquierda. Meba irá mostrando los conceptos de dos en dos, y cambiará el color del triángulo para que el alumno sea consciente que el sistema avanza.

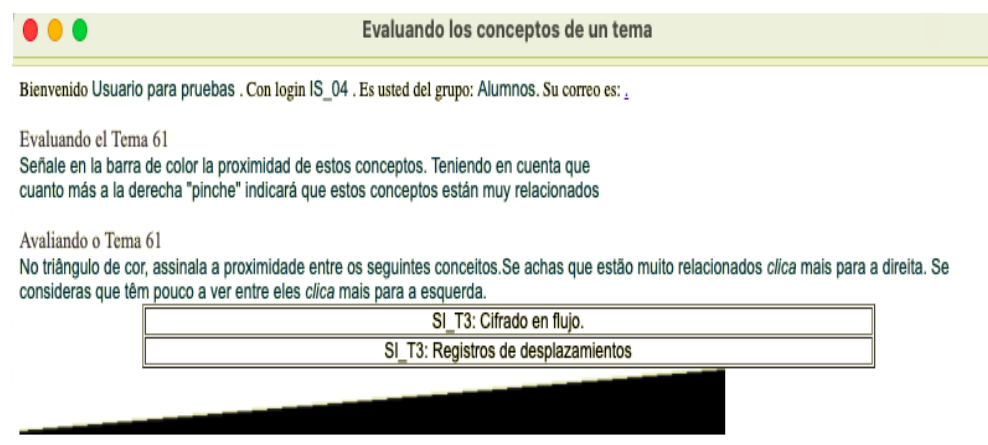

Fig. 4. Pantalla evaluando la proximidad de dos conceptos.

El servidor Meba exporta hacia el exterior la matriz de similaridad correspondiente a cada una de las evaluaciones.

En la Fig. 5, se muestra un ejemplo de una matriz de similaridad. En ella, se puede ver que es el resultado de un usuario llamado "seguridad04"; que es una matriz de similaridad de conceptos; que se hace para 4 nodos, es decir, 4 conceptos; que los valores que almacena son decimales; que el valor mínimo es 0 y el máximo 768, en consecuencia si dos conceptos son muy similares tendrán un valor de similaridad próximo al 768 , mientras que dos conceptos alejados tendrán un valor próximo a 0; que la matriz que se presenta es triangular, solo aparecen la mitad de los datos, pues es una matriz simétrica; y finalmente, los valores de similaridad de cada par de conceptos.

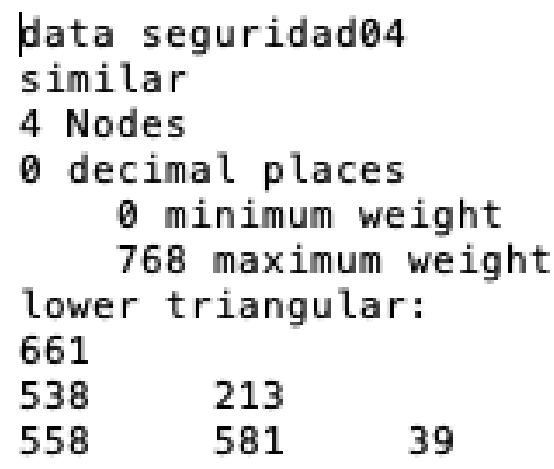

Fig. 5. Matriz de similaridad del alumno "seguridad04"

Al igual que Meba exporta las matrices de similaridad, también es posible exportar las matrices de distancia, cuyos valores serían los opuestos a los de similaridad, es decir, si dos pares de conceptos en el ejemplo que estamos mostrando en la Fig. 5 tienen un valor de similaridad de 661 si se hubiera exportado una matriz de distancia el valor sería 107 (768-661), y así con el resto. 


\subsection{Caso de Estudio}

El caso de estudio que se ha realizado sólo participaron 28 alumnos de los 32 posibles, pues el resto, aunque están matriculados en la asignatura no han asistido regularmente a clase, por ello, no pudieron participar en las dos "evaluaciones de proximidad". Sendas evaluaciones se corresponden con la toma de datos antes de la explicación del tema y luego posteriormente a dicha explicación. Ambas evaluaciones nos han permitido poder diferenciar el antes y después de la explicación y poder "medir" cómo cambian las redes medias de proximidad de los alumnos a partir de la clase magistral del profesor.

Además, de las 28 redes se tuvieron que eliminar 3 en la primera toma de datos y 2 en la segunda debido a la coherencia (es decir, a la falta de la misma) que tenía cada una de las redes. Esto se debe a que según (Schvaneveldt, 1989b) aquellas matrices cuyo valor de coherencia (la coherencia es un valor entre -1 y 1) sea menor que 0,15 no debe ser tenida en cuenta pues se supone que el encuestado lo ha contestado al azar.

Por tanto, en este trabajo se han definido los alumnos con los login SI_2020_01 al SI 2020 32. Estos alumnos se han agrupado en el grupo SI Antes y el grupo SI Despues, para que el servidor Meba pueda ofrecer la evaluación de proximidad antes y después de la explicación. Además, se ha definido el tema "Cistografía moderna" al que se le han asignado los conceptos: Cifrado en flujo, Cifrado en bloque, Registros de desplazamientos y Generadores de secuencias. Con todo ello, se pudo hacer las dos tomas de datos, antes y después de la explicación cuyos resultados se analizan en el apartado siguiente.

\section{Resultados}

Los resultados de las dos evaluaciones de proximidad de conceptos se procesaron con la teoría de Redes Asociativas Pathfinder (RAP), para lo cual se utilizó el programa JPathfinder, (Schvaneveldt, R., 2017) que permite calcular las redes medias de un grupo de matrices, la similaridad, la RAP, etc.

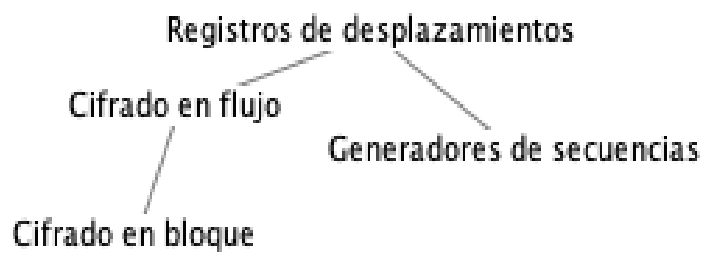

Fig. 6. RAP de la red media de los alumnos antes de la explicación

En ambas tomas de datos participaron 28 alumnos. Sin embargo, las matrices válidas para trabajar han sido 25 en la primera toma Fig. 6 y 26 en la segunda, esas cinco matrices que faltan fueron eliminadas por el equipo de investigación por la falta de coherencia Fig. 7 que tenían. A partir de las 25 matrices de la primera toma de datos se calcula red media de los alumnos que mostramos en la Fig. 6.

Como se puede observar se trata de una red totalmente lineal, con nodos centrales Registros de desplazamiento y Cifrado en flujo, pero la información que aporta es muy poca.

Igualmente, con las 26 matrices de similaridad resultantes de la segunda toma de datos, que tuvo lugar después de la explicación del tema, se obtiene la red media de los alumnos que mostramos en la Fig 7. En esta RAP se puede ver que hay un nodo nuclear (Casas, 2002), (Arias, Contreras, Hidalgo, y Martín, 2019) , "Registros de desplazamientos". 
En la Fig. 8 mostramos la RAP del profesor, en la que el nodo nuclear "Registros de desplazamientos" coincide con el nodo nuclear de la RAP de la red media de los alumnos después de la explicación, lo cual nos hace prever buenos resultados respecto a la similaridad de las redes asociativas pahtfinder que estamos buscando validar. La diferencia visual que se puede apreciar entre la Fig. 7 y la Fig. 8 es que el concepto "generadores de secuencia" además de estar unido al nodo nuclear, también está unido con el concepto de "control de flujo".

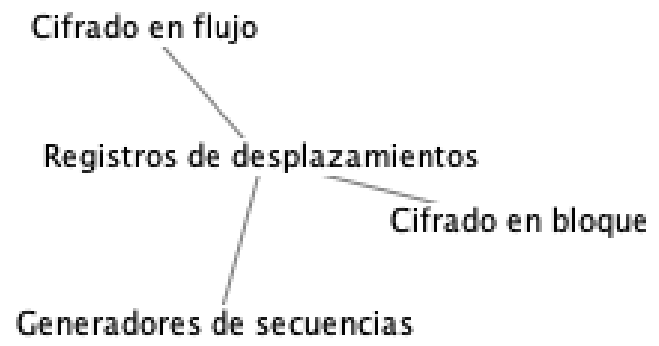

Fig. 7. RAP de la red media de los alumnos después de explicación

Por tanto, el profesor puede observar que la relación estrecha que hay entre "generadores de secuencia" y "control de flujo" no ha debido quedar bien explicado, pues si bien el cifrado en flujo necesita un generador de secuencia para poder llevar a cabo el cifrado, es algo que no aparece reflejado en la red media de los alumnos. Todo ello, permite al profesor saber en tiempo real cómo se están aproximando las redes cognitivas de los alumnos a la del profesor una vez que está teniendo lugar la puesta en marcha del proceso de enseñanza-aprendizaje.

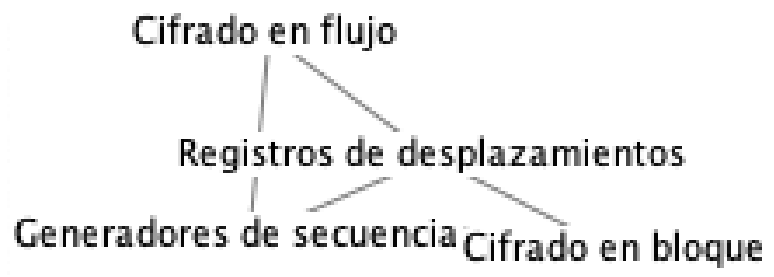

Fig. 8. Red Asociativa Pathfinder del profesor.

Los resultados del análisis de JPathfinder para la coherencia se muestran en la Fig. 9, en ella podemos ver el gran aumento de coherencia que hay en la red media de los alumnos después de la explicación frente a la que había antes de la misma, concretamente pasa de 0,32 a 0,60 . Esto es un resultado esperable, pues después de la explicación es más fácil para los alumnos ser capaces de reflejar la similaridad de los conceptos de forma más coherente que antes, entre otras razones porque es algo que conocen mejor con lo cual es más fácil reflejar el conocimiento. 
Vol. 9 | Investigación Cualitativa en Ciencias Sociales: Avances y Desafíos

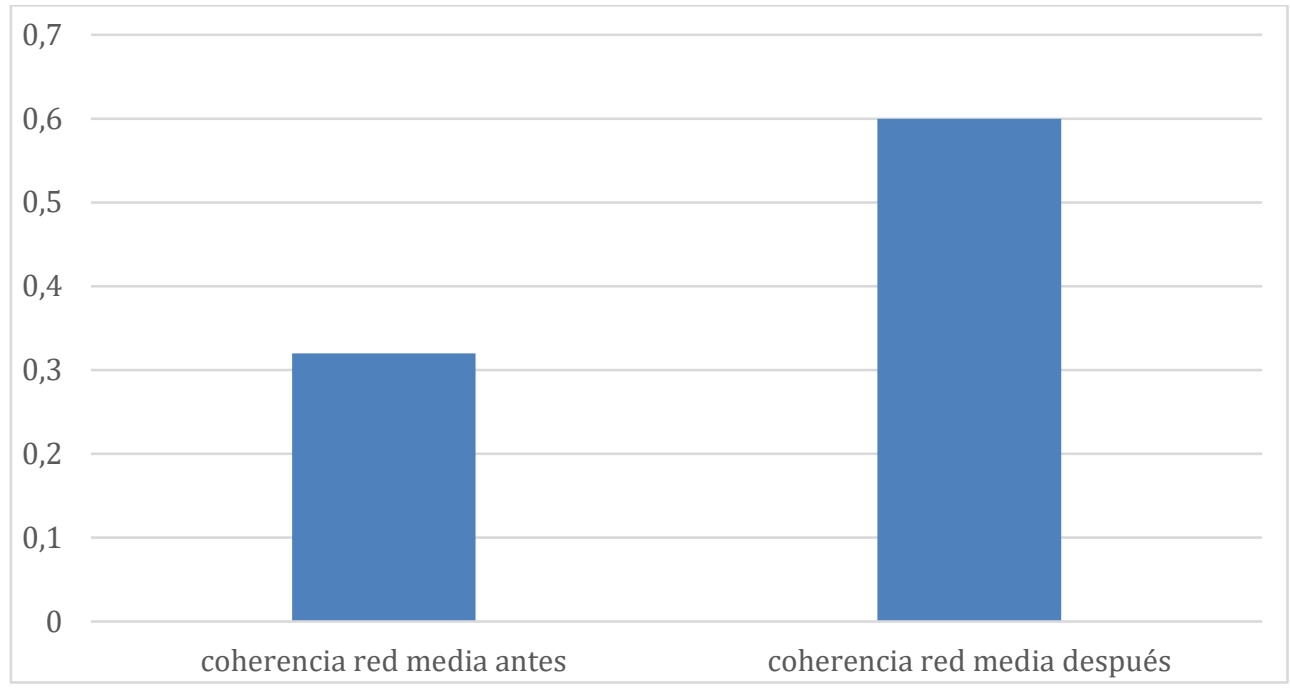

Fig. 9. Comparativa de la coherencia de las redes medias de los alumnos antes y después de la explicación.

El resumen del análisis de similaridad antes y después de la explicación de las RAP de las redes medias de los alumnos frente a la del profesor se puede ver en la Fig. 10, en la cual observamos cómo la similaridad ha pasado de 0,25 a 0,75 que es un gran incremento de similaridad, esta similaridad se muestra reflejado en las Fig. 6, Fig. 7 y Fig. 8 como hemos comentado antes, donde la Fig. 7 y la Fig. 8 tienen el mismo nodo nuclear.

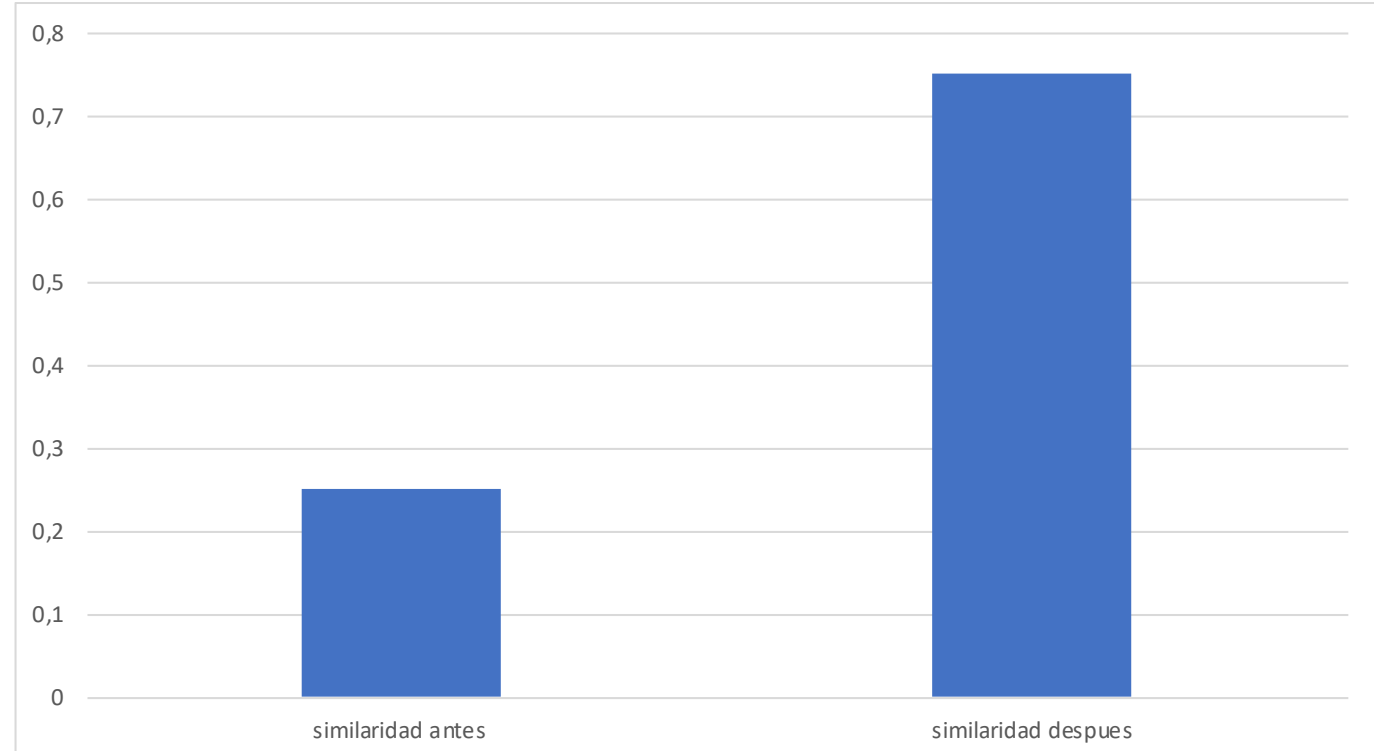

Fig. 10. Similaridad de las redes medias de los alumnos antes y después de la explicación frente a la red del profesor.

\section{Conclusiones}

El objetivo principal de esta investigación es ofrecer al profesor una herramienta que le aporte información en tiempo real del conocimiento que están recibiendo sus alumnos mientras imparte un determinado tema. Hemos elegido un estudio de 4 conceptos concretos de uno de los temas y se han realizado test de similaridad a los alumnos sobre el conocimiento de estos conceptos antes y después de la explicación del tema de estudio. 
El dato más relevante que se obtiene es la diferencia entre la red de la Fig. 7 y la Fig. 8 donde como hemos indicado se aprecia la falta de conexión que debería haber entre el nodo de "cifrado en flujo" y el nodo de "generadores de secuencia" con lo cual el profesor puede concluir que esa relación de proximidad de estos dos conceptos no ha sido transmitida adecuadamente.

Además, hemos podido comprobar cómo las redes de después de la instrucción son más coherentes que las previas, se ha pasado de 0,32 a 0,6. E igualmente, ha ocurrido con la similaridad de las redes medias de los alumnos frente a la red del profesor que se ha pasado de un 0,25 antes de la explicación a un 0,75 después de la misma. Por tanto, podemos indicar que las RAP nos pueden ayudar a detectar las posibles "lagunas" que puedan tener lugar en la transmisión del conocimiento del profesor a los alumnos.

En consecuencia, concluimos que hemos conseguido un método con el que conocer la evolución el proceso de enseñanza/aprendizaje de sus alumnos, siendo además de fácil utilización por parte de los profesores, ya que rápidamente y en tiempo real podrán evaluar el aprendizaje de los alumnos.

Nuestra idea, como equipo de investigación es continuar con la creación del software que integre y automatice de forma integral el proceso aquí descrito.

\section{Agradecimientos}

A todas las personas implicadas directamente en esta investigación, especialmente a los estudiantes de Seguridad de la Información, que han dedicado su tiempo para aportarnos los datos.

\section{Referencias}

Arias, J. (2017). Web del Servidor Meba. Proyecto II. http://meba.unex.es

Arias, J., Contreras, J. A., Hidalgo, V. y Martín, R. (2019). Medición de la aproximación de las redes medias de los alumnos a la red del profesor durante la instrucción usando Redes Asocitivas Pathfinder. Ciaiq2019, 3, 694-697.

Arias, J. (2008). Evaluación de la calidad de Cursos Virtuales: Indicadores de Calidad y construcción de un cuestionario a medida. Aplicación al ámbito de asignaturas de Ingeniería Telemática. Universidad de Extremadura.

Ausubel D.P, Novak, J.D. y Hanesian, H. (1978). Psicología Educativa: Un punto de vista cognoscitivo. México: Trillas.

Casas, L. M. (2002). El estudio de la estructura cognitiva de alumnos a través de Redes Asociativas Pathfinder. Aplicaciones y posibilidades en Geometría. In Universidad de Extremadura. https://www.mendeley.com/catalogue/bf6b7ef7-1f3a-3f4b-9f5dee48cc4b3f66/?utm_source=desktop\&utm_medium=1.19.4\&utm_campaign=open_catalog\&u serDocumentld=\%7Bי7317ee9-5aae-49a0-91e4-d92f2135a12d\%7D

Casas, L. M. y Luengo, R. (2013). La Teoría de los Conceptos Nucleares y su aplicación en la investigación en Didáctica de las Matemáticas. UNIÓN. Revista Iberoamericana de Educación Matemática, 34, 9-36.

Centro Criptológico Nacional. Gobierno de. (2017). Generadores de secuencia. Guía de Seguridad de Las TIC. https://www.ccn-cert.cni.es/series-ccn-stic/800-guia-esquema-nacional-deseguridad/513-ccn-stic-807-criptologia-de-empleo-en-el-ens/file.html

Contreras, J. Á., Arias, J., Luengo, R. y Casas, L. M. (2015). Índices de Nuclearidad (Completo y Reducido), como aportación a la Teoría de Conceptos Nucleares. RISTI - Revista Iberica de Sistemas e Tecnologias de Informacao, E4, 16-34. https://doi.org/10.17013/risti.e4.16-34 
Hidalgo, V., Arias, J., Casas, L., Luengo, R. y Castillo, A. (2008). Redes cognitivas de alumnos y Red de la Ciencia: Similaridad. CISCI 2008 - Septima Conferencia Iberoamericana En Sistema, Cibernetica e Informatica 5to SIECI 2008, 3er Simposium Internacional En Comunicacion Del Conocimiento y Conferencias, CCC 2008 - Memorias, 1, 68-73. http://www.iiis.org/cds2008/cd2008csc/SIECI2008/PapersPdf/X336OI.pdf

Martinez, F.M. (2016). Criptosistemas de cifrado en flujo basados en matrices triangulares con multiples bloques [Universidad de Alicante]. In Universidad de Alicante (Vol. 1). https://rua.ua.es/dspace/bitstream/10045/54318/1/tesis_francisco_miguel_martinez_perez.pd $f$

Piaget, J. (1978). Lecturas de la Psicología del niño. In La evolución intelectual entre la adolescencia y la edad adulta.

Schvaneveldt, R. W. (1989a). Pathfinder associative networks (Ablex Publ). Ablex Publishing.

Schvaneveldt, R. W. (1989b). Pathfinder Associative Networks: Studies in Knowledge Organization.

Schvaneveldt, R. W. (2017). http://interlinkinc.net/JPathfinder.htm. Schvaneveldt, R. (2017) JPathfinder. Las Cruces, NM USA. http://interlinkinc.net/JPathfinder.htm 